\title{
Editorial
}

\section{Recent Development in Fixed-Point Theory, Optimization, and Their Applications}

\author{
Jinlu Li, ${ }^{1}$ Chong Li, ${ }^{2}$ Ngai-Ching Wong, ${ }^{3}$ and Jen-Chih Yao ${ }^{4}$ \\ ${ }^{1}$ Department of Mathematics, Shawnee State University, Portsmouth, OH 45662, USA \\ ${ }^{2}$ Department of Mathematics, College of Science, Zhejiang University, Hangzhou, Zhejiang 310027, China \\ ${ }^{3}$ Department of Applied Mathematics, National Sun Yat-Sen University, Kaohsiung 804, Taiwan \\ ${ }^{4}$ Center for Fundamental Science, Kaohsiung Medical University, Kaohsiung 807, Taiwan \\ Correspondence should be addressed to Jinlu Li; jli@shawnee.edu
}

Received 10 June 2014; Accepted 10 June 2014; Published 19 June 2014

Copyright (C) 2014 Jinlu Li et al. This is an open access article distributed under the Creative Commons Attribution License, which permits unrestricted use, distribution, and reproduction in any medium, provided the original work is properly cited.

This special volume was originally conceived to provide authors and readers a publication to present the most recent advances in the study of applications of various fixed point theorems. Since 1909, when Luitzen Brouwer proved the first fixed point theorem named after him, fixed point theory has played very important roles in many different fields. We can find a lot of demonstrations in optimization theory, approximation theory, differential equations, variational inequalities, complementary problems, equilibrium theory, game theory, economics theory, and so forth.

Fixed point theorems are developed for single-valued or set-valued mappings of metric spaces, topological vector spaces, posets and lattices, Banach lattices,.... Among the themes of fixed point theory, the topic of approximation of fixed points of mappings is particularly important because it is useful for proving the existence of fixed points of mappings. It can be applied to prove the solvability of optimization problems, differential equations, variational inequalities, and equilibrium problems.

Due to the importance of and the high volume of active research in the nonlinear analysis and optimization and, in particular, many new tools in studying them involving fixed point approximations, it is worthwhile to publish a special issue on this topic to highlight the recent advances in this field.

The selection of the papers included in this volume, which has been based on a strict international peer review procedure, contains a representative list of papers with newfangled results which covers the different topics considered in our original proposal.

In the papers in this special issue, the following topics are discussed.

(i) General nonlinear analysis theory and applications:

"Applications of Bregman-Opial property to Bregman nonspreading mappings in Banach spaces," by E. Naraghirad et al.;

"Application of extended homotopy analysis method to the two-degree-of-freedom coupled van der pol-duffing oscillator" by Y. H. Qian et al.;

"The hierarchical minimax inequalities for setvalued mappings," by Y.-C. Lin, and C.-T. Pang.

(ii) Variational inequalities, optimization problems, complementarity problems, and equilibrium problems:

"Generalized equilibrium problems related to Ky Fan inequalities" by I. Roventa;

"Strong convergence theorems for solutions of equilibrium problems and common fixed points of a finite family of asymptotically nonextensive nonself mappings" by L. Zhang et al.;

"Bregman f-projection operator with applications to variational inequalities in banach spaces" by C.-T. Pang et al. 
(iii) Approximation of fixed and common fixed points of mappings in ordered Banach and metric spaces:

"A new approach to fixed point results in triangular intuitionistic fuzzy metric spaces" by $\mathrm{N}$. Hussain et al.;

"Applications of fixed point theorems to generalized saddle points of bifunctions on chaincomplete posets" by J. Li et al.;

"Fixed point results for various $\alpha$-admissible contractive mappings on metric-like spaces" by S. Al-Mezel et al.;

"n-tupled coincidence point theorems in partially ordered metric spaces for compatible mappings" by S. Dalal et al.

(iv) Approximation of solutions of differential equations, optimization problems, variational inequalities, complementarity problems:

"Hybrid algorithms for solving variational inequalities, variational inclusions, mixed equilibria, and fixed point problems" by L.-C. Ceng et al.;

"Second-order Regularity estimates for singular Schrodinger equations on convex domains" by X. Tao;

"Multistep hybrid iterations for systems of generalized equilibria with constraints of several problems" by L.-C. Ceng et al.

(v) Convergent iterative process for mappings in Banach spaces:

"Iterative schemes for finite families of maximal monotone operators based on resolvents" by $\mathrm{L}$. Wei and R. Tan;

"A generalized inexact Newton method for inverse eigenvalue problems" by W. Shen;

"Hierarchical fixed point problems in uniformly smooth Banach spaces" by L.-C. Ceng et al.

\section{Acknowledgments}

The editors of this special issue would like to express their gratitude to the authors who have submitted their papers for consideration and to the many expert referees who were involved in the reviewing process and provided us with invaluable help.

Jinlu Li

Chong $\mathrm{Li}$

Ngai-Ching Wong

Jen-Chih Yao 


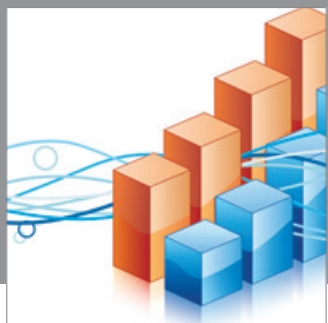

Advances in

Operations Research

mansans

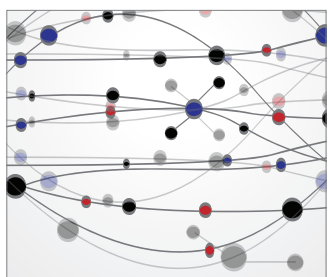

The Scientific World Journal
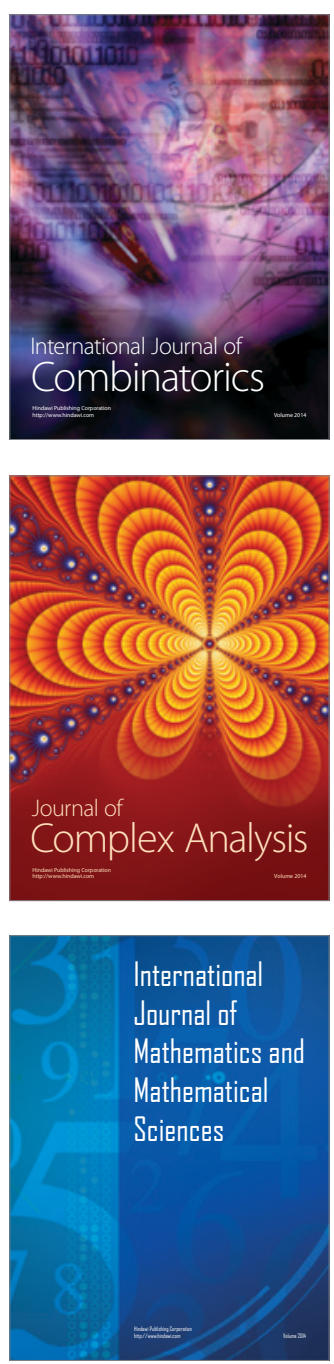
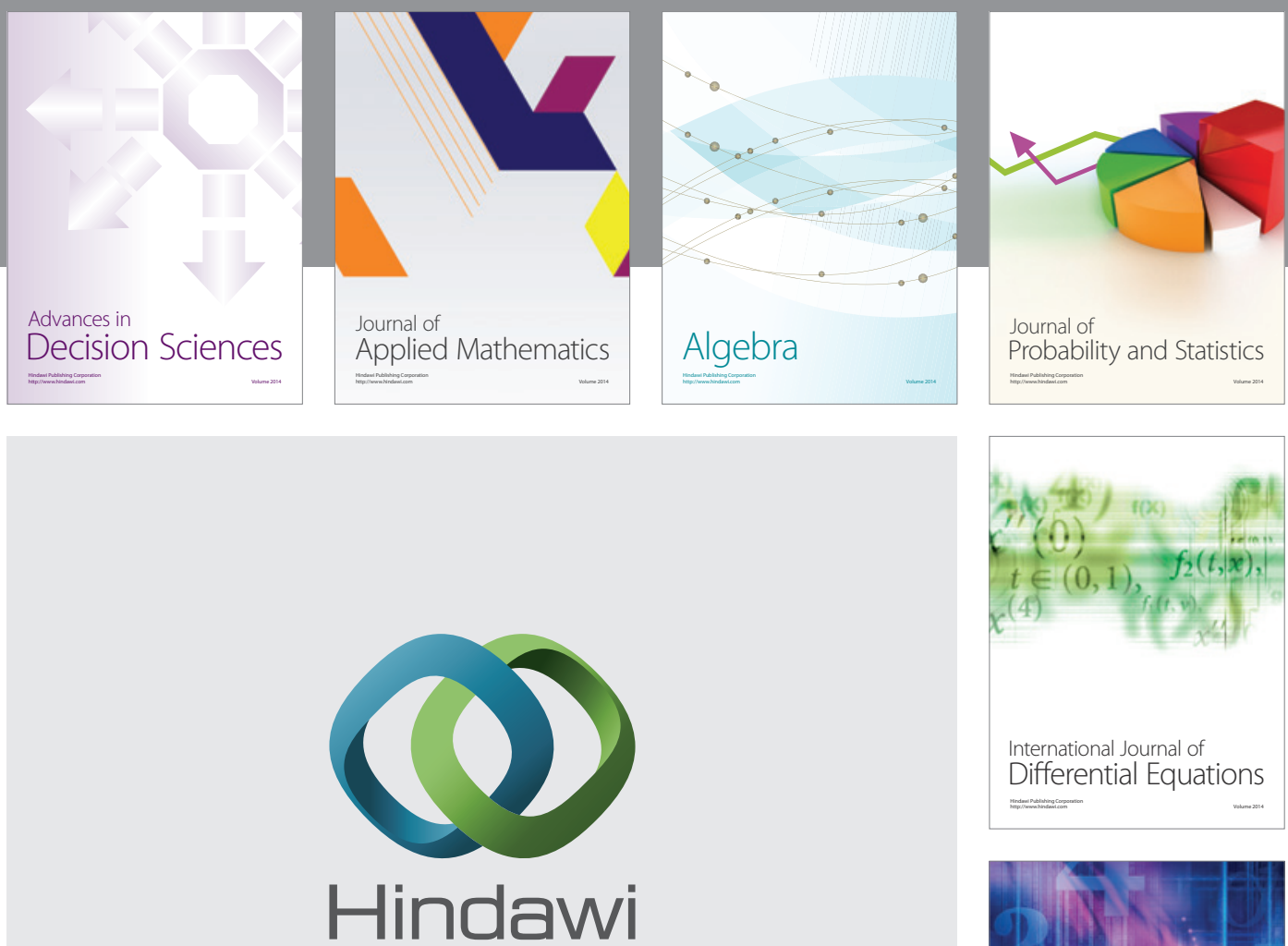

Submit your manuscripts at http://www.hindawi.com
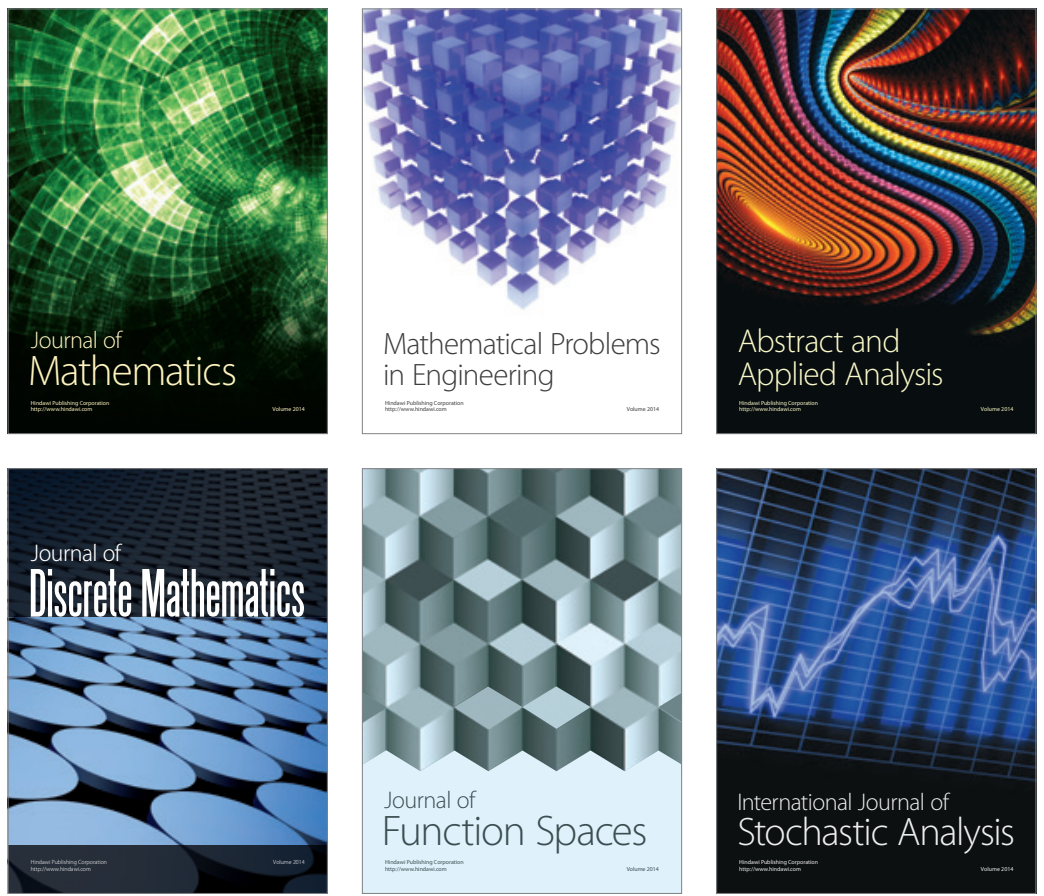

Journal of

Function Spaces

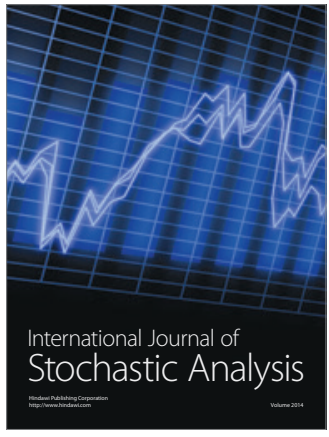

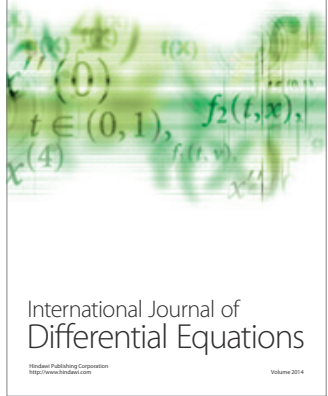
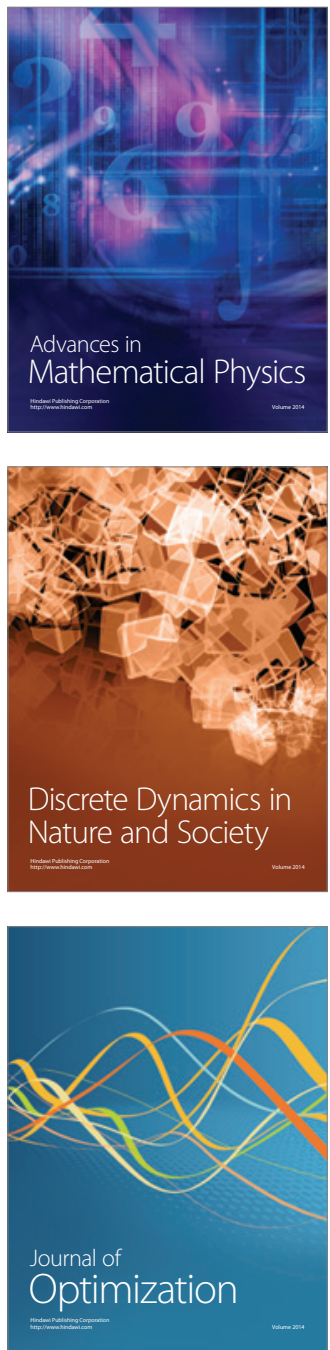